\title{
On a theorem of S. Tanaka
}

By

W. A. HARRIS, Jr.

During the Symposium on Functional Equations held at Osaka University, January 19-20, 1966 Sen-ichiro Tanaka presented some interesting results concerning solutions of a class of nonlinear difference equations. In the ensuing discussion, the question of a possible extension of these results was raised. It is the purpose of this note to answer this question in the negative through the construction of a counter example.

For the scalar case, Tanaka's results can be phrased in the following manner.

Consider the nonlinear difference equation

$$
y(x+1)=y^{n} f(x, y),
$$

where $n$ is an integer greater than one and $f(x, y)$ is an analytic function of $x$ and $y$ for $|x|>r,|\arg x|<\beta,|y|<\delta$.

Let

$$
f(x, y)=\sum_{k=0}^{\infty} f_{k}(x) y^{k}
$$

be the expansion of $f(x, y)$ in powers of $y$. We assume that the $f_{k}(x)$ are analytic for $|x|>r,|\arg x|<\beta$ and admit the asymptotic expansions

$$
f_{k}(x) \cong \sum_{j=0}^{\infty} f_{k j} x^{-j}
$$

as $x$ approaches infinity through the region $|\arg x|<\beta$.

Further, we assume

Received April 28, 1966.

Communicated by M. Hukuhara.

School of Mathematics, University of Minnesota and Research Institute for Mathematical Sciences, Kyoto University. U.S.-Japan Cooperative Science Program, NSF Grant GF-214. 


$$
f_{00} \neq 0 .
$$

Tanaka has shown that there exists a nonlinear transformation

$$
y=u[1+p(x, u)]
$$

which transforms the difference equation

$$
y(x+1)=y^{n} f(x, y)
$$

into the difference equation

$$
u(x+1)=u^{n} f_{00} .
$$

The transformation (1) is analytic for $x \in R,|u|<\delta^{\prime}$, where $R$ is a suitable subregion of $|x|>r,|\arg x|<\beta$ extending to infinity. Further, we have the asymptotic expansion

$$
p(x, u) \cong \sum_{j \geq 1} \sum_{k \geq 0} p_{j k} u^{j} x^{-k}
$$

as $|u|+|x|^{-1}$ approaches zero, $x \in R,|u|<\delta^{\prime}$.

We are concerned with the following question.

Question. Can the same type of results be obtained when $n$ is a rational number by allowing the nonlinear transformation (1) to contain fractional powers?

Counter example.

There is no formal transformation of the form

$$
y=u[1+p(u)]
$$

where

$$
p(u)=\sum_{k=1}^{\infty} p_{k} u^{k \omega}
$$

with $\alpha$ a positive rational number, which transforms the diffrence equation

$$
y(x+1)=y^{3 / 2}(1+y)
$$

into the difference equation

$$
u(x+1)=u^{3 / 2} .
$$


Proof.

The existence of the transformation (2) is equivalent to a solution of the equation

$$
1+p\left(u^{3 / 2}\right)=[1+p(u)]^{3 / 2}[1+u+u p(u)] .
$$

Write

$$
[1+p(u)]^{3 / 2}=1+\frac{3}{2} p(u)+h(p(u)) .
$$

Then equation (3) becomes

$$
\begin{aligned}
p\left(u^{3 / 2}\right)=\frac{3}{2} p(u)+u & +\frac{5}{2} u p(u)+h(p(u))+\frac{3}{2} u p(u)^{2} \\
& +u h(p(u))+u p(u) h(p(u)) .
\end{aligned}
$$

Case 1: $\alpha>1$.

Suppose there exists a formal solution $p(u)=\sum_{k=1}^{\infty} p_{k} u^{k \alpha}$ with $\alpha>1$. Then the following order relations hold $: p\left(u^{3 / 2}\right)=O\left(u^{3 \alpha / 2}\right)=$ $o(u) ; p(u)=O\left(u^{\alpha}\right)=o(u)$; and $h(p(u))=O\left(p(u)^{2}\right)=O\left(u^{2 \alpha}\right)=o(u)$. Thus every term in equation (4) is of order $o(u)$ except the term $u$. Hence there can be no formal solution of this form in this case.

Case 2: $0<\alpha \leqq 1$.

If there is a solution of equation (4) of the form $\sum p_{k} u^{k \alpha}$ with rational $\alpha, 0<\alpha \leqq 1$, there exists an integer $q \geqq 1$ and a formal solution of the form

$$
p(u)=\sum_{j=1}^{\infty} \bar{p}_{j} u^{j / q} .
$$

We shall now establish three properties of the coefficients of the formal series (5) under the assumption that this series is a formal solution of equation (4).

\section{Property 1.}

$$
\bar{p}_{1}=\bar{p}_{2}=\cdots=\bar{p}_{q-1}=0, \bar{p}_{q} \neq 0 .
$$

This is a simple computation using the order relations for the various terms. In fact, $\bar{p}_{q}=-2 / 3$. 


\section{Property 2.}

If the equation (4) has a solution of the form $\sum_{k=1}^{\infty} \bar{p}_{k} u^{k / q}$, then this solution can be written in the form $\sum_{k=1}^{\infty} \bar{p}_{2 k} u^{2 k / q}$.

Consider $k$ such that $\bar{p}_{k} \neq 0$. The term $p\left(u^{3 / 2}\right)$ will contribute the nonzero term $\bar{p}_{k} u^{3 k / 2 q}$ to the left side of equation (4). The terms on the right side of equation (4) are of the form $u^{i / q}$. Hence, if $\bar{p}_{k} \neq 0$, then $k=2 i$ for some $i$.

\section{Property 3.}

$q$ must be an even integer.

From properties 1 and $2, \bar{p}_{q} \neq 0$ and hence $q=2 i$ for some $i$. Since $q$ is an integer, we may write

$$
q=2^{m} q^{\prime}
$$

where $q^{\prime}$ is an odd integer. By using property $2 m$-times, we are led to the conclusion that equation (4) has a solution of the form

$$
p(u)=\sum_{k=1}^{\infty} \widehat{p}_{k} u^{k / g^{\prime}}
$$

If $q^{\prime}=1$, then using property 2 again shows that there is a solution of the form $p(u)=\sum \hat{p}_{2 k} u^{2 k}$ which is impossible by Case 1 with $\alpha=2$.

If $q^{\prime}>1$, then property 3 shows that $q^{\prime}$ must be even which is a contradiction.

Hence, there is no solution of equation (4) of the indicated form and the counter example has been established.

\section{REFERENCE}

[1] S. Tanaka, On the general solution of nonlinear difference equations, Publ. Res. Inst. Math. Sci. Kyoto Univ. Ser. A to appear. 